\title{
517.
}

\section{SUR LES SURFACES DIVISIBLES EN CARRÉS PAR LEURS COURBES DE COURBURE ET SUR LA THÉORIE DE DUPIN.}

[From the Comptes Rendus de l'Académie des Sciences de Paris, tom. Lxxiv. (JanvierJuin, 1872), pp. 1445-1449.]

Solent $\Theta$ une fonction arbitraire de $h, k ; x, y, z$ des fonctions de $h, k$ telles que

$$
\begin{aligned}
& 2 \Theta \frac{d^{2} x}{d h d k}-\frac{d \Theta}{d h} \frac{d x}{d k}-\frac{d \Theta}{d k} \frac{d x}{d h}=0, \\
& 2 \Theta \frac{d^{2} y}{d h d k}-\frac{d \Theta}{d h} \frac{d y}{d k}-\frac{d \Theta}{d k} \frac{d y}{d h}=0, \\
& 2 \Theta \frac{d^{2} z}{d h d k}-\frac{d \Theta}{d h} \frac{d z}{d k}-\frac{d \Theta}{d k} \frac{d z}{d h}=0,
\end{aligned}
$$

et que, de plus,

$$
\frac{d x}{d h} \frac{d x}{d k}+\frac{d y}{d h} \frac{d y}{d k}+\frac{d z}{d h} \frac{d z}{d k}=0
$$

en éliminant $h, k$, on a, entre $x, y, z$, l'équation $V=0$ d'une surface. Je dis que les. équations $h=$ const., $k=$ const. déterminent les deux systèmes des courbes de courbure de cette surface, et, de plus, que cette surface est divisible en carrés par ses courbes. de courbure.

En effet, les équations donnent

ce qui implique

$$
\Theta \frac{d}{d k}\left[\left(\frac{d x}{d h}\right)^{2}+\left(\frac{d y}{d h}\right)^{2}+\left(\frac{d z}{d h}\right)^{2}\right]-\frac{d \Theta}{d k}\left[\left(\frac{d x}{d h}\right)^{2}+\left(\frac{d y}{d h}\right)^{2}+\left(\frac{d z}{d h}\right)^{2}\right]=0,
$$

$$
\left(\frac{d x}{d h}\right)^{2}+\left(\frac{d y}{d h}\right)^{2}+\left(\frac{d z}{d h}\right)^{2}=\Theta H
$$


où $H$ est fonction de $h$ seulement; et l'on trouve de même

$$
\left(\frac{d x}{d k}\right)^{2}+\left(\frac{d y}{d k}\right)^{2}+\left(\frac{d z}{d k}\right)^{2}=\Theta K
$$

où $K$ est fonction de $k$ seulement; donc en écrivant, comme à l'ordinaire,

$$
d x^{2}+d y^{2}+d z^{2}=E d h^{2}+2 F d h d k+G d k^{2},
$$

cette expression se réduit à

$$
d x^{2}+d y^{2}+d z^{2}=\Theta\left(H d h^{2}+K d k^{2}\right),
$$

ce qui fait voir que la surface est divisible en carrés par les courbes

$$
h=\text { const. }, \quad k=\text { const. }
$$

Les équations donnent aussi

$$
\left|\begin{array}{ccc}
\frac{d x}{d h}, & \frac{d y}{d h}, & \frac{d z}{d h} \\
\frac{d x}{d k}, & \frac{d y}{d k}, & \frac{d z}{d k} \\
\frac{d^{2} x}{d h d k}, & \frac{d^{2} y}{d h d k}, & \frac{d^{2} z}{d h d k}
\end{array}\right|=0 ;
$$

et, cela étant, l'équation différentielle des courbes de courbure se réduit, comme je vais le montrer, à $d h d k=0$; on a donc $h=$ const., $k=$ const., pour les équations des courbes de courbure de la surface.

Pour cela, en considérant $x, y, z$ comme des fonctions données de $h$, $k$, j'écris, comme à l'ordinaire,

$$
\frac{d x}{d h}=a, \quad \frac{d x}{d k}=a^{\prime}, \quad \frac{d^{2} x}{d h^{2}}=\alpha, \quad \frac{d^{2} x}{d h d k}=\alpha^{\prime}, \quad \frac{d^{2} x}{d k^{2}}=\alpha^{\prime \prime},
$$

et de même $b, b^{\prime}, \beta, \beta^{\prime}, \beta^{\prime \prime}$, et $c, c^{\prime}, \gamma, \gamma^{\prime}, \gamma^{\prime \prime}$ pour les coefficients différentiels de $y$ et $z$ respectivement. J'écris aussi

$$
\begin{array}{lll}
A=b c^{\prime}-b^{\prime} c, & B=c a^{\prime}-c^{\prime} a, & C=a b^{\prime}-a^{\prime} b, \\
E=a^{2}+a^{\prime 2}+a^{\prime \prime 2}, & F=a a^{\prime}+b b^{\prime}+c c^{\prime}, & G=a^{\prime 2}+b^{\prime 2}+c^{\prime 2} .
\end{array}
$$

L'équation différentielle des courbes de courbure est

$$
\left|\begin{array}{lll}
d x, & d y, & d z \\
A, & B, & C \\
d A, & d B, & d C
\end{array}\right|=0
$$

Le premier terme de ce déterminant est $d x(B d C-C d B)$, savoir:

$$
\begin{aligned}
& \left(a d h+a^{\prime} d k\right)\left\{B\left[\left(a \beta^{\prime}-b \alpha^{\prime}+b^{\prime} \alpha-a^{\prime} \beta\right) d h+\left(a \beta^{\prime \prime}-b \alpha^{\prime \prime}+b^{\prime} \alpha^{\prime}-a^{\prime} \beta^{\prime}\right) d k\right]\right. \\
& \left.-C\left[\left(c \alpha^{\prime}-a \gamma^{\prime}+a^{\prime} \gamma-c^{\prime} \alpha\right) d h+\left(c \alpha^{\prime \prime}-a \gamma^{\prime \prime}+a^{\prime} \gamma^{\prime}-c^{\prime} \alpha^{\prime}\right) d k\right]\right\},
\end{aligned}
$$

C. VIII. 
ce qui se réduit tout de suite à

$$
\begin{aligned}
\left(a d h+\alpha^{\prime} d k\right)\{ & {\left[a\left(A \alpha^{\prime}+B \beta^{\prime}+C \gamma^{\prime}\right)-a^{\prime}(A \alpha+B \beta+C \gamma)\right] d h } \\
- & {\left.\left[a\left(A \alpha^{\prime \prime}+B \beta^{\prime \prime}+C \gamma^{\prime \prime}\right)-a^{\prime}\left(A \alpha^{\prime}+B \beta^{\prime}+C \gamma^{\prime}\right)\right] d k\right\} }
\end{aligned}
$$

en formant les expressions analogues du second et du troisième terme, et en prenant la somme, l'équation devient

$$
\begin{aligned}
& {\left[E\left(A \alpha^{\prime}+B \beta^{\prime}+C \gamma^{\prime}\right)-F(A \alpha+B \beta+C \gamma)\right] d h^{2} } \\
+ & {\left[E\left(A \alpha^{\prime \prime}+B \beta^{\prime \prime}+C \gamma^{\prime \prime}\right)-G(A \alpha+B \beta+C \gamma)\right] d h d k } \\
+ & {\left[F\left(A \alpha^{\prime \prime}+B \beta^{\prime \prime}+C \gamma^{\prime \prime}\right)-G\left(A \alpha^{\prime}+B \beta^{\prime}+C \gamma^{\prime}\right)\right] d k^{2}=0 }
\end{aligned}
$$

ou, ce qui est la même chose,

$$
\left|\begin{array}{ccc}
d k^{2}, & -d h d k, & d h^{2} \\
E, & F, & G \\
A \alpha+B \beta+C \gamma, & A \alpha^{\prime}+B \beta^{\prime}+C \gamma^{\prime}, & A \alpha^{\prime \prime}+B \beta^{\prime \prime}+C \gamma^{\prime \prime}
\end{array}\right|=0 ;
$$

celle-ci est l'équation différentielle des courbes de courbure d'une surface quand les coordonnées $x, y, z$ d'un point de la surface sont données comme fonctions de deux paramètres $h, k$.

En supposant $F=0$, l'équation se réduit à

$$
\begin{aligned}
& \left(A \alpha^{\prime}+B \beta^{\prime}+C \gamma^{\prime}\right)\left(E d h^{2}-G d k^{2}\right) \\
+ & {\left[\left(A \alpha^{\prime \prime}+B \beta^{\prime \prime}+C \gamma^{\prime \prime}\right) E-(A \alpha+B \beta+C \gamma) G\right] d h d k=0 ; }
\end{aligned}
$$

et en supposant de plus $A \alpha^{\prime}+B \beta^{\prime}+C \gamma^{\prime}=0$, l'équation se réduit simplement à $d h d k=0$; mais cette équation $A \alpha^{\prime}+B \beta^{\prime}+C \gamma^{\prime}=0$, savoir

$$
\left|\begin{array}{ccc}
a, & b, & c \\
a^{\prime}, & b^{\prime}, & c^{\prime} \\
\alpha^{\prime}, & \beta^{\prime}, & \gamma^{\prime}
\end{array}\right|=0
$$

ou

$$
\left|\begin{array}{ccc}
\frac{d x}{d h}, & \frac{d y}{d h}, & \frac{d z}{d h} \\
\frac{d x}{d k}, & \frac{d y}{d k}, & \frac{d z}{d k} \\
\frac{d^{2} x}{d h d k}, & \frac{d^{2} y}{d h d k}, & \frac{d^{2} z}{d h d k}
\end{array}\right|=0,
$$

et aussi $F=0$, subsistent dans le cas actuel; et nous avons ainsi $d k d h=0$ pour équation différentielle des courbes de courbure. 
On vérifie sans peine les équations fondamentales, en prenant $\Theta=h-k$,

$$
\begin{aligned}
& -(c-a)(a-b) x^{2}=a(a+h)(a+k) \\
& -(a-b)(b-c) y^{2}=b(b+h)(b+k) \\
& -(b-c)(c-a) z^{2}=c(c+h)(c+k)
\end{aligned}
$$

ce qui donne les courbes de courbure de l'ellipsoïde $\frac{x^{2}}{a}+\frac{y^{2}}{b}+\frac{z^{2}}{c}=1$; l'ellipsoïde étant, comme on sait, une surface divisible en carrés par des courbes de courbure; mais je n'ai pas encore cherché d'autres solutions.

Je remarque que l'équation pour $x$ peut s'écrire sous la forme

$$
\frac{d}{d h}\left(\frac{1}{\Theta} \frac{d x}{d k}\right)+\frac{d}{d k}\left(\frac{1}{\Theta} \frac{d x}{d h}\right)=0
$$

donc, en posant

$$
-\frac{d}{d h}\left(\frac{1}{\Theta} \frac{d x}{d k}\right)=\frac{d}{d k}\left(\frac{1}{\Theta} \frac{d x}{d h}\right)=\frac{d^{2} \Omega}{d h d k}
$$

on trouve

$$
\frac{d x}{d h}=\Theta \frac{d \Omega}{d h}, \quad \frac{d x}{d k}=-\Theta \frac{d \Omega}{d k}
$$

ce qui donne

$$
\frac{d}{d k}\left(\Theta \frac{d \Omega}{d h}\right)+\frac{d}{d h}\left(\Theta \frac{d \Omega}{d k}\right)=0
$$

équation pour $\Omega$ de la même forme que celle pour $x$.

On déduit une démonstration très-simple du théorème de Dupin. En considérant comme auparavant $(x, y, z)$ comme des fonctions données de $(h, k)$, le point $(x, y, z)$ sera situé sur une surface, et les conditions pour que les courbes de courbure soient $h=$ const., $k=$ const. seront

$$
\begin{gathered}
\frac{d x}{d h} \frac{d x}{d k}+\frac{d y}{d h} \frac{d y}{d k}+\frac{d z}{d h} \frac{d z}{d k}=0 \\
\left|\begin{array}{ccc}
\frac{d x}{d h}, & \frac{d y}{d h}, & \frac{d z}{d h} \\
\frac{d x}{d k}, & \frac{d y}{d k}, & \frac{d z}{d k} \\
\frac{d^{2} x}{d h d k}, & \frac{d^{2} y}{d h d k}, & \frac{d^{2} z}{d h d k}
\end{array}\right|=0
\end{gathered}
$$

Cela étant, en introduisant un troisième paramètre $l$, soient $h, k, l$ des fonctions données de $(x, y, z)$, ou réciproquement $(x, y, z)$ des fonctions données de $(h, k, l)$. On 
a ici les trois systèmes de surfaces $h=$ const., $k=$ const., $l=$ const., et les conditions pour que ces surfaces se coupent orthogonalement peuvent s'écrire sous la forme

$$
\begin{aligned}
& \frac{d x}{d k} \frac{d x}{d l}+\frac{d y}{d k} \frac{d y}{d l}+\frac{d z}{d k} \frac{d z}{d l}=0, \\
& \frac{d x}{d l} \frac{d x}{d h}+\frac{d y}{d l} \frac{d y}{d h}+\frac{d z}{d l} \frac{d z}{d h}=0, \\
& \frac{d x}{d h} \frac{d x}{d k}+\frac{d y}{d h} \frac{d y}{d k}+\frac{d z}{d h} \frac{d z}{d k}=0 .
\end{aligned}
$$

On a donc

$$
\frac{d x}{d l}: \frac{d y}{d l}: \frac{d z}{d l}=\frac{d y}{d h} \frac{d z}{d k}-\frac{d z}{d h} \frac{d y}{d k}: \frac{d z}{d h} \frac{d x}{d k}-\frac{d x}{d h} \frac{d y}{d k}: \frac{d x}{d h} \frac{d y}{d k}-\frac{d y}{d h}: \frac{d z}{d h}
$$

Pour abréger, j’écris

et de même

$$
\frac{d x}{d h} \frac{d x}{d k}+\frac{d y}{d h} \frac{d y}{d k}+\frac{d z}{d h} \frac{d z}{d k}=[h . k], \ldots
$$

$$
\frac{d x}{d h} \frac{d^{2} x}{d k d l}+\frac{d y}{d h} \frac{d^{2} y}{d k d l}+\frac{d z}{d h} \frac{d^{2} z}{d k d l}=[h . k l], \ldots .
$$

Les conditions données sont ainsi

$$
[k . l]=0, \quad[l . h]=0, \quad[h . k]=0 ;
$$

en différentiant ces équations par rapport à $h, k, l$ respectivement, on obtient

donr

$$
\begin{aligned}
& {[k \cdot l h]+[l . h k]=0,} \\
& {[l \cdot k h]+[h . k l]=0,} \\
& {[h \cdot k l]+[k \cdot l h]=0 ;}
\end{aligned}
$$

$$
[h . k l]=0, \quad[k . l h]=0, \quad[l . k h]=0 .
$$

Mais l'équation $[h . k]=0$ et l'équation $[l . h k]=0$, en substituant dans celle-ci les valeurs de $\frac{d x}{d l}, \frac{d y}{d l}, \frac{d z}{d l}$, sont précisément les conditions pour que la surface $l=$ const. soit coupée par les autres surfaces selon ses courbes de courbure: donc le théorème. 